\title{
Recently Cleared Concept
}

National Cancer Institute

\section{Source}

National Cancer Institute. Recently Cleared Concept. NCI Thesaurus. Code C19473.

Document type (funding opportunity): a PA or RFA not requiring clearance at the $\mathrm{NCl}$ Board of Scientific Advisors 